J. Clin. Chem. Clin. Biochem.

Vol. 26, 1988, pp. 123-133

(C) 1988 Walter de Gruyter \& Co.

Berlin - New York

\title{
$D-\left[{ }^{3} \mathrm{H}\right]$ Glucosamine Labelling of Epidermal and Dermal Glycosaminoglycans in Cultured Human Skin
}

\author{
By J.H. Poulsen, I. M. Jensen
}

Department of Clinical Chemistry, University of Aarhus, Aarhus Kommunehospital, Aarhus, Denmark and

\section{U. Petersen}

Department of Otorhinolaryngology, University of Aarhus, Aarhus Kommunehospital, Aarhus, Denmark

(Received June 27, 1986/July 27/December 21, 1987)

Summary: An in vitro model is presented for the study of glycosaminoglycans in human skin. The synthesis of six glycosaminoglycan species in both dermis and epidermis was measured by $D-\left[{ }^{3} \mathrm{H}\right]$ glucosamine labelling. Punched biopsies (epidermis + entire dermis) of $3 \mathrm{~mm}$ in diameter were cultured at $37^{\circ} \mathrm{C}$ in $5 \%$ carbon dioxide- $95 \%$ air. When the label was added $18 \mathrm{~h}$ after explantation, the incorporation started immediately, and for all glycosaminoglycans the time-dependent incorporation was linear for $16 \mathrm{~h}$. The experimental variation was minimized by expressing the measurements in epidermis "per explant" and in dermis "per mg of wet explant". A ratio to dermal hydroxyproline did not improve the precision. Most of the variation arose "before" isolation and separation of the glycosaminoglycans. The labelled products were macromolecules and were converted to small molecules by chondroitinase $\mathrm{ABC}+$ heparinase. The total incorporation in dermis was $2 \frac{1}{2}$ times higher than in epidermis. Hyaluronic acid was the predominant synthesized product in dermis, and hyaluronic acid and heparan sulphate were the predominant products in epidermis. The proportions (\%) in dermis/epidermis were as follows: hyaluronic acid, 61/44; heparan sulphate, 18/31; dermatan sulphate, 5/8; chondroitin 4/6-sulphate, 10/7 and heparin-like glycosaminoglycan, 1/2. The same species were also demonstrated as native constituents of uncultured human skin. Hyaluronic acid and dermatan sulphate predominated in dermis, whereas no single species predominated in epidermis. Their concentrations in uronic acid equivalents per mg of wet skin (pmol/mg of epidermis + dermis) were as follows in dermis/epidermis: hyaluronic acid, 243/0.48; heparan sulphate, 22/0.44; dermatan sulphate, 170/0.56; chondroitin 4/6-sulphate, 72/0.50; and heparin-like glycosaminoglycan, $5 / 0.22$. Thus, only $0.4 \%$ of the in vivo synthesized glycosaminoglycan was present in epidermis.

\section{Introduction}

Glycosaminoglycans, mainly associated with proteins in proteoglycans, are important constituents of cell surfaces and extracellular matrix (1). As glycosaminoglycans are believed to be involved in the control of cellular differentiation and tissue development (2, 3 ), in vitro studies of glycosaminoglycan in wound healing and cancer will probably attract more attention in the future. For such studies cell culture is not always suitable. Without normal sociocellular rela- tionships cell culture may not only result in distortion of cellular phenotype and karyotype (3), but may also give misleading information about the glycosaminoglycan turnover. Thus, without physical contact between epidermis and dermis the epidermal synthesis of hyaluronic acid in cultured pig skin decreases considerably (4). Conversely, cultured corneal fibroblasts also produce markedly less glycosaminoglycan in the absence than in the presence of corneal epithelium (6, $6,7)$. 
Supported by the work of King and his associates (4, 8) we developed a reliable method for the study of glycosaminoglycan synthesis in cultured human skin by means of $D-\left[{ }^{3} \mathrm{H}\right]$ glucosamine labelling. We believe that this method meets an acute need to study the synthesis of glycosaminoglycan in vitro, under conditions where "normal" mesenchymal-epithelial relationships as well as cellular environments are preserved.

The characteristics distinguishing this method from others can be summarized as follows: the method needs only very small biopsies and is therefore suitable for studies on human skin; in fact all studies were performed on human skin. In the model the synthesis of 6-7 glycosaminoglycan species is followed in both dermis and epidermis. Hence, not only major synthesis products like hyaluronic acid and heparan sulphate are measured, but reliable results are also obtained for minor products such as dermatan sulphate, heparin and chondroitin 4/6-sulphate. In addition we also measured the content of in vivo synthesized glycosaminoglycans in dermis and epidermis. By comparing these contents with the synthesis figures above, we were able to assess the relative rate at which each of these glycosaminoglycans is replaced in the tissues.

\section{Materials and Methods}

\section{Materials}

Keratan sulphate type I from bovine cornea, heparan sulphate from bovine kidney, and keratanase from Pseudomonas were obtained from Seikagaku Co., Japan. Hyaluronic acid grade III-S from human umbilical cord, hyaluronidase from Streptomyces, chondroitin sulphate type A (= chondroitin 4-sulphate) from whale cartilage, chondroitin sulphate type B (= dermatan sulphate) from porcine skin, chondroitinase AC from Arthrobacter aurescens, chondroitinase ABC from Proteus vulgaris, papain type IV, deoxyribonuclease I type III from bovine pancreas, ribonuclease-A type III-A from bovine pancreas, and bovine serum albumin, crystallized and lyophilised, were obtained from Sigma, USA. Crude heparinase from Flavobacterium heparinum and heparin sodium salt USP XIX grade were generous gifts from Novo, Denmark. $D-\left[{ }^{3} \mathrm{H}\right]$ glucosamine hydrochloride was from Amersham International Ltd., UK. Alcian Blue was from Bio-Rad laboratories, UK. Cetyltrimethylammoniumbromide was from Koch-Light Laboratories, UK. Ethylene-diaminetetra-acetic acid and $L$-cysteine hydrochloride were analytical grade chemicals from Merck, FRG. Sepharose 6B was from Pharmacia Fine Chemicals, Sweden. Minimum essential medium modified with Earle's salts with $20 \mathrm{mmol} / \mathrm{l}$ HEPES, $L$-glutamine, and foetal calf serum (batch number 29023132) were from Flow Laboratories, UK. Penicillin and streptomycin were prepared as a frozen solution at our hospital dispensary. Pico-Fluor 15 was obtained from United Technologies Packard, USA.

\section{Reagents}

\section{Alcian Blue solution}

$2.5 \mathrm{~g} / \mathrm{l}$ of alcian Blue and $12.17 \mathrm{~mol} / 1$ of methanol in 0.83 $\mathrm{mmol} / \mathrm{l}$ of acetic acid.

\section{Acetic acid solution}

$50 \mathrm{mmol} / \mathrm{l}$ acetate buffer, $\mathrm{pH}$ 5.5.

\section{Carrier glycosaminoglycan}

Post mortem skin, $5 \times 5 \mathrm{~mm}^{2}$, treated with $1 \mathrm{~mol} / 1$ calcium chloride for $1 \mathrm{~h}$ at $37^{\circ} \mathrm{C}$, was deepithelialized, cut into pieces, washed 3 times in water and defatted in ether + acetone $(3+1$ by vol.) at $37^{\circ} \mathrm{C}$ for $1 \mathrm{~h}$. The defatted dermis was disintegrated in a Thomas Wiley Micro Mill (Arthur H. Thomas Co., Philadelphia, USA), defatted for further $24 \mathrm{~h}$ in methanol + chloroform $(3+1$ by vol.) at ambient temperature and dried under vacuum. Fifty $\mathrm{mg}$ portions of dried dermis were digested at $67^{\circ} \mathrm{C}$ for $7 \mathrm{~h}$ by $50 \mu \mathrm{l}$ of "papain solution" (see below) in 1000 $\mu l$ of "ethylene-diaminetetra-acetic acid solution" (see below). The resulting incubates were pooled and stored at $-20^{\circ} \mathrm{C}$. The glycosaminoglycan concentration of the pool was measured in uronic acid equivalents on a cetyltrimethylammonium bromide precipitate (section VIII).

\section{Cetyltrimethylammonium bromide solution}

$189 \mathrm{nmol} / 1$ of cetyltrimethylammonium bromide in $20 \mathrm{nmol} / \mathrm{l}$ sodium chloride.

\section{Culture medium}

Minimum essential medium modified with Earle's salts, containing $20 \mathrm{nmol} / \mathrm{l}$ of HEPES, $4 \mathrm{mmol} / 1 \mathrm{~L}$-glutamine, $44 \mathrm{mmol} / \mathrm{l}$ of sodium bicarbonate, foetal calf serum, volume fraction 0.1 , $10^{4}$ units per 1 of penicillin and $100 \mathrm{mg} / \mathrm{l}$ of streptomycin.

\section{Ethylene-diaminetetra-acetic acid solution}

An aqueous solution containing $50 \mathrm{mmol} / \mathrm{l}$ ethylene-diaminetetra-acetic acid and $5 \mathrm{mmol} / 1$ of $L$-cysteine hydrochloride, adjusted to $\mathrm{pH} 7.3$.

\section{Glycosaminoglycan degrading enzyme solution}

Concentrated solutions of chondroitinase AC, chondroitinase $A B C$, heparinase, keratanase or combinations of these enzymes in tris buffer and Streptomyces hyaluronidase in acetate buffer were added in volumes of $1 \mu \mathrm{l}$.

\section{Hyaluronidase buffer}

$20 \mathrm{mmol} / \mathrm{l}$ of sodium acetate, and $150 \mathrm{mmol} / \mathrm{l}$ of sodium chloride, $\mathrm{pH} 6.0$.

\section{Papain-solution}

An aqueous solution containing $24 \mathrm{~g} / 1$ of papain.

\section{Tris buffer-deoxyribonuclease/ribonuclease solution}

$50 \mathrm{mmol} / \mathrm{l}$ of tris buffer, $\mathrm{pH} 7.4$, containing $35 \mathrm{mmol} / 1$ of sodium acetate, $1.7 \mathrm{mmol} / \mathrm{l}$ of calcium acetate, $50 \mathrm{mmol} / 1$ of sodium chloride, $4.1 \mathrm{mmol} / 1$ of magnesium chloride, $0.1 \mathrm{~g} / \mathrm{l}$ of bovine albumin, $2.5 \mathrm{~g} / \mathrm{l}$ of deoxyribonuclease and $2.5 \mathrm{~g} / \mathrm{l}$ of ribonuclease.

\section{Tris buffer solution}

$50 \mathrm{mmol} / \mathrm{l}$ of tris buffer, $\mathrm{pH} 7.4$, containing $35 \mathrm{mmol} / 1$ of sodium acetate, $" 1.7 \mathrm{mmol} / \mathrm{l}$ of calcium acetate, $50 \mathrm{mmol} / 1$ of sodium chloride and $0.1 \mathrm{~g} / \mathrm{l}$ of bovine albumin. .: 


\section{Specimens of skin}

All skin materials were of human origin and included epidermis and the entire dermis. The tissue culture experiments were performed on biopsies stored at $4{ }^{\circ} \mathrm{C}$ for less than $2 \mathrm{~h}$ in $5 \%$ carbon dioxide- $95 \%$ oxygen-treated "culture-medium". Post mortem skin, stored at $-20^{\circ} \mathrm{C}$, was used for measurement of native in vivo synthesized glycosaminoglycans and production of "carrier glycosaminoglycan".

\section{Tissue culture technique with $D-\left[{ }^{3} \mathrm{H}\right]$ glucosamine labelling}

Punched skin disks with a diameter of $3 \mathrm{~mm}$ (= explant) were placed on siliconized lens papers, $15 \times 15 \mathrm{~mm}^{2}$, with epidermis upward. Two grafts of explant-bearing lens papers were floated on $5 \mathrm{ml}$ of "culture-medium" in a plastic petri dish with a diameter of $5 \mathrm{~cm}$. The petri dishes were incubated in $5 \%$ carbon dioxide $-95 \%$ air at $37^{\circ} \mathrm{C}$. After $18 \mathrm{~h}$ of incubation $1.85 \mathrm{MBq}$ $(50 \mu \mathrm{Ci})$ of $D\left[{ }^{3} \mathrm{H}\right]$ glucosamine hydrochloride was added to each dish, and the culture was continued for further $16 \mathrm{~h}$. The incorporation was terminated by momentary freezing of the explants on dry-ice. The explants were kept at $-20^{\circ} \mathrm{C}$ until they were analysed.

V. Isolation of labelled glycosaminoglycan from dermis and epidermis

The explants were thawed, blotted on filter paper and weighed (= ivet weight of the explant). After treatment in $1 \mathrm{~mol} / 1 \mathrm{calcium}$ chloride for $1 \mathrm{~h}$ at $37^{\circ} \mathrm{C}$ epidermis was separated from dermis as an intact layer.

Dermis was washed for $10 \mathrm{~min}$ in $2 \mathrm{ml}$ of water, cut into pieces, and dissolved in $400 \mu \mathrm{l}$ of "ethylene-diaminetetra-acetic acid solution". Five $\mu$ l of "papain-solution" was added and the sample was digested for $4 \mathrm{~h}$ at $67^{\circ} \mathrm{C}$, then sonicated for $15 \mathrm{~s}$, and digested overnight.

Epidermis was digested at $67^{\circ} \mathrm{C}$ overnight by $5 \mu$ l of "papainsolution" in $250 \mu \mathrm{l}$ of "ethylene-diaminetetra-acetic acid solution", sonicated for $10 \mathrm{~s}$, and finally digested for further $4 \mathrm{~h}$. The digested dermis and epidermis were then treated alike. After centrifugation for $5 \mathrm{~min}$ at $1900 \mathrm{~g}$ the supernatant was transferred to a plastic tube. "Carrier-glycosaminoglycan" with a uronic acid content of $45 \mathrm{nmol}$ (about $200 \mu \mathrm{l}$ ) and $1800 \mu \mathrm{l}$ of $20 \mathrm{mmol} / \mathrm{l}$ sodium chloride were added. $\mathrm{pH}$ was adjusted to 11 and $300 \mu \mathrm{l}$ of "cetyltrimethylammonium bromide" was added.

Then the first precipitation was initiated: after $2 \mathrm{~h}$ at ambient temperature the glycosaminoglycans were collected by centrifugation at $1900 \mathrm{~g}$ for $25 \mathrm{~min}$. This centrifugation was repeated after a washing procedure with $600 \mu \mathrm{l}$ of "acetic acid solution". The precipitate was redissolved by successive addition of $40 \mu \mathrm{l}$ of $n$-propanol, $200 \mu \mathrm{l}$ of $n$-propanol (volume fraction 0.6 ) in water-and $600 \mu \mathrm{l}$ of ethanol. Fifty $\mu \mathrm{l}$ of $1.84 \mathrm{~mol} / 1$ sodium acetate was added, and the solution was left overnight in an ice-water bath. After centrifugation at $1900 \mathrm{~g}$ for $15 \mathrm{~min}$ the precipitate was redissolved in $50 \mu \mathrm{l}$ of "tris buffer-deoxyribonuclease/ribonuclease-solution" and incubated for $5 \mathrm{~h}$ at $37^{\circ} \mathrm{C}$. One thousand $\mu \mathrm{l}$ of $20 \mathrm{mmol} / \mathrm{l}$ sodium chloride and $200 \mu \mathrm{l}$ of "cetyltrimethylammonium bromide-solution" were added to the digest.

Then the second precipitation was started: apart from the washing procedure which was performed with water, the second precipitation was similar to the first one. The final precipitate was redissolved in $50 \mu \mathrm{l}$ of "tris buffer-solution" and processed as described in the following section VI.

\section{Electrophoresis of labelled glycosaminoglycan}

Electrophoresis was performed at $\mathrm{pH} 6.7$ in $0.1 \mathrm{~mol} / \mathrm{l}$ phosphate buffer on strips of cellulose acetate (Schleicher and Schüll, FRG), $25 \times 180 \mathrm{~mm}^{2}$. Twenty $\mu \mathrm{l}$ of the glycosaminoglycan preparation from the preceding section $\mathrm{V}$ was applied as a strip, $2 \mathrm{~cm}$ from the margin of the cathode wick, and processed for $60 \mathrm{~min}$ at $10 \mathrm{~V} / \mathrm{cm}$. The remaining $30 \mu \mathrm{l}$ of the glycosaminoglycan-solution was incubated for $60 \mathrm{~min}$ at $37^{\circ} \mathrm{C}$ with $0.20 \mathrm{U}$ of chondroitinase AC. After addition of $7.5 \mathrm{nmol}$ uronic acid equivalents of heparan sulphate an electrophoresis of $20 \mu \mathrm{l}$ of the digested glycosaminoglycan-solution was performed. The strips were stained for $5 \mathrm{~min}$ in "Alcian Blue-solution", $40 \mathrm{ml}$ per strip, dipped in $50 \mathrm{ml} / 1$ acetic acid, and washed twice for $3 \mathrm{~min}$ in $50 \mathrm{ml} / 1$ acetic acid, $40 \mathrm{ml}$ per strip. The labelled glycosaminoglycans were recovered in three electrophoretic bands (b, $c$ and $d$ in fig. 1) detected on the cellulose acetate by the Alcian Blue-stained carrier glycosaminoglycans. Guided by these 3 bands 6 fractions ( $a, b, c, d, e$, and $f$ in fig. 1) were cut out from each cellulose acetate electrophoresis, transferred to counting tubes and dissolved overnight in $3 \mathrm{ml}$ of concentrated acetic acid. The labelled fractions were then counted as described in the following section VII.

VII. Liquid scintillation counting of labelled glycosaminoglycan-species

After addition of $16 \mathrm{ml}$ of Pico-Fluor to the acetic acid solution of dissolved cellulose acetate and labelled glycosaminoglycan the tube was counted in a Tri-Carb 2450 liquid scintillation counter (Packard, USA) for 4 min or up to 10000 counts. All the radioactivity was located in fractions $b, c$ and $d$. No significant activity was ever measured in fractions $a, e$ and $f$. The counts of the f-fractions were used as blank values and were subtracted from the counts of the remaining fractions. The radioactivity of the individual glycosaminoglycan-species were calculated as shown in table 1 using the "B-formulas". Originating from samples of $20 \mu \mathrm{l}$, this radioactivity represented $2 / 5$ of the activity in the whole explant. To present the actual count figures we expressed the epidermal results as counts/min $\cdot 20 \mu \mathrm{l}$ (reduced to counts/min in tables and figures) and the dermal results as counts/min $\cdot 20 \mu \mathrm{l} \cdot \mathrm{mg}$ wet explant (reduced to counts/ $m i n \cdot m g$ in tables and figures).

Tab. 1. Calculation of radioactivity or densitometric scanning area of glycosaminoglycan species separated by electrophoresis and enzyme pretreatment. Depending on the separation procedure used, calculations were performed by two sets of formulae, $A$ and $B$. The origin of the raw data used in the formulae can be deduced from the "Roman numeral-letter" combinations. The Roman numeral indicates whether the sample was electrophoresed without (I) or after treatment with chondroitinase AC (II) or chondroitinase ABC (III). The letter indicates whether the measurement (radioactivity or scanning area) originates from the electrophoretic fractions b, c, or d (see fig. 1).

\begin{tabular}{lll}
\hline & Formulae \\
\cline { 2 - 3 } Glycosaminoglycans & A & B \\
\hline Hyaluronic acid & Id-IIId & Id-IId \\
Heparan sulphate & IIIc & IIc \\
Dermatan sulphate & IIb-IIIb & IIb \\
Heparin & IIIb & Ib-IIb \\
Chondroitin 4/6-sulphate & Ib-IIb & Ic-IIc \\
Compound X & Ic-IIIc & IId \\
Compound Y & IIId & \\
\hline
\end{tabular}


VIII. Concentration measurement of in vivo synthesized glycosaminoglycans isolated from uncultured skin

Fifty and $57 \mathrm{mg}$ of dried defatted dermis and epidermis were prepared and digested by papain (sections II and V). Using a scaled-up version of the method described in section $\mathrm{V}$, without adding "carrier-glycosaminoglycan", the native glycosaminoglycans were isolated in a "tris buffer solution". By measuring the uronic acid content of this solution (section $\mathrm{X}$ ) the total tissue concentration of glycosaminoglycans was calculated. On the remaining material the percentages of the glycosaminoglycan-species were established as described below. The product between the total concentration and these percentages gave the uronic acid concentration of the individual glycosaminoglycanspecies. The glycosaminoglycans were fractionated by electrophoresis as described in section VI. An amount of glycosaminoglycan equivalent to $5 \mathrm{nmol}$ of uronic acid was electrophoresed without and after treatment for $60 \mathrm{~min}$ at $37^{\circ} \mathrm{C}$ with $0.013 \mathrm{U}$ of chondroitinase AC, $0.013 \mathrm{U}$ of chondroitinase AC $+0.008 \mathrm{U}$ of chondroitinase $\mathrm{ABC}$, or $0.013 \mathrm{U}$ of chondroitinase $\mathrm{AC}+0.008 \mathrm{U}$ chondroitinase $\mathrm{ABC}+3.6 \mu \mathrm{g}$ of heparinase, respectively, per nmol of uronic acid. The wet strips were stained with Alcian Blue and scanned at $620 \mathrm{~nm}(0.1 \mathrm{~mm}$ slit) in a Cliniscan (Helena Laboratories, USA). The densitometric scanning areas of the individual glycosaminoglycan-species were calculated as described in table 1 using the "A-formulas". Preliminary studies showed that these areas correlated linearily with the amount of 'glycosaminoglycan ( $<5 \mathrm{nmol}$ of uronic acid equivalents); but unfortunately the glycosaminoglycanspecies took up stain with different affinities. The scan-areas were therefore corrected for differences in stainability before they were used for calculating the skin concentrations of the individual species. The uptake of Alcian Blue per mol uronic acid by heparin, hyaluronic acid, heparan sulphate, chondroitin 4-sulphate, and dermatan sulphate were related to each other as 1.8 to 0.7 to 1.0 to 1.0 to 1.0 . The concentrations of epidermal and dermal glycosaminoglycan-species were expressed in uronic acid equivalents as pmol/mg of wet explant.

IX. Characterization of chondroitinase AC suscep= tible material in band $c$

Glycosaminoglycans from $50 \mathrm{mg}$ of dry dermis were isolated, incubated with 1 Unit of Streptomyces hyaluronidase in $60 \mu \mathrm{l}$ of "hyaluronidase buffer" for $2 \mathrm{~h}$ at $60^{\circ} \mathrm{C}$. The incubate was reprecipitated, electrophoresed with and without previous chondroitinase treatment, stained with Alcian Blue and scanned.

$X$. Measurement of uronic acid, hexosamine and hydroxyproline

Methods described elsewhere were used for colorimetric measurement of uronic acid (10) and hexosamine (10). The content of hydroxyproline in dermis was measured by the Hypronosticon ${ }^{\circledR}$ test from Organon (Holland). Fifty $\mu$ l of papain-digested dermis mixed with $450 \mu \mathrm{l}$ of water was analysed as described in the manufacturer's instructions.

\section{Results}

Substrate specificity of keratanase, heparinase, chondroitinase $A C$, and chondroitinase $\mathrm{ABC}$

The enzymes used were initially tested against authentic preparations of glycosaminoglycans. The enzymes in question were incubated for $60 \mathrm{~min}$ at $37^{\circ} \mathrm{C}$ with hyaluronic acid, chondroitin 4-sulphate, der- matan sulphate, heparin, heparan sulphate and keratan sulphate type I; they were all present at uronic acid or hexosamine concentrations of $30 \mathrm{nmol}$ in $60 \mu \mathrm{l}$ "tris buffer solution". Digested and undigested substrates were processed by cellulose acetate electrophoresis, and the effect of the enzymes was measured by the reduction they produced in the 'Alcian Blue positive material on the electrophoresis strip.

Chondroitinase AC (0.3 unit) only digested hyaluronic acid and chondroitin 4-sulphate. Digestion by chondroitinase AC did not change the mobility or stainability of dermatan sulphate from pörcine skin, when electrophoresed on cellulose acetate. Chondroitinase $\mathrm{ABC}$ ( 0.1 unit) in addition digested dermatan sulphate. When the authentic glycosaminoglycans were incubated with $10 \mu \mathrm{g}$ of heparinase (in fact a crude preparation of heparinase and heparitinases), all except keratan sulphate and dermatan sulphate were completely digested. Dermatan sulphate was not totally digested until the amount of heparinase was increased to $48 \mu \mathrm{g}$. Keratan sulphate resisted incubation with $90 \mu \mathrm{g}$ of heparinase. Keratanase $(0.12$ and 0.24 unit) was only active against keratan sulphate, but unveiled $21 \%$ of resistant material with an $18 \%$ higher electrophoretic mobility than the original keratan sulphate fraction. This material may represent polysulphated keratan sulphate, which resists degradation by keratanase according to the manufacturer, and Muir \& Hardingham (1).

\section{Labelled glycosaminoglycans in epidermis and dermis}

Glycosaminoglycan from dermis or epidermis, gives the same 3 electrophoretic fractions $b, c$ and $d$ (fig. 1). Dermal glycosaminoglycans could therefore be used as electrophoretic markers of labelled glycosaminoglycans from both epidermis and dermis. The labelled fractions were cut out from the cellulose acetate strips, solubilized in acetic acid and mixed with scintillation liquid (sections VI and VII). The solutions were homogeneous and clear, but contained solubilized cellulose acetate and Alcian Blue. As shown in figure 2 the counting efficiency did not change with increasing concentrations of both these components.

It was shown that the labelled, in vitro synthesized glycosaminglycans also separated into three distinct bands, with low activities (less than $26 \%$ of the total) in the electrophoretic areas between the b, c and dbands (activity $/ \mathrm{cm}^{2}$ ). There was no significant radioactivity outside the three b-, c-, and d-fractions marked by the carrier-glycosaminoglycans, not even at the origin or in the neighbouring fractions d, e, 


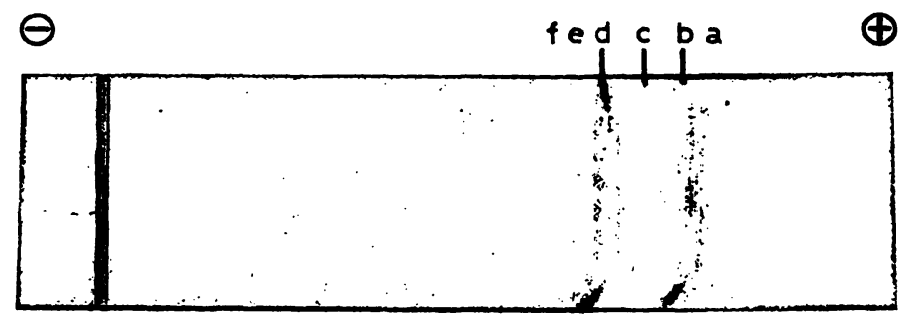

Origin

Fig. 1. Alcian Blue-stained cellulose acetate electrophoresis of carrier-glycosaminoglycan. Carrier-glycosaminoglycan of dermal origin, used for the isolation of labelled glycosaminoglycans, was separated into three bands, b, c and $d$, as described in section VI. These bands as well as a narrow strip anodic to band $b(=$ fraction $a)$ and two strips cathodic to band $d$ ( = fraction e and $f$ ) were cut out for radioactivity measurements. All six fractions had equal dimensions and constituted an adjoining piece of the cellulose acetate strip. Fraction $f$ was used for establishing a blank value.

and $\mathrm{f}$. Fraction $\mathrm{f}$, used for establishing a blank value, thus contained less than $1 \%$ of the radioactivity in each of the b-, c-, and d-bands.

Removal of labelled material from the electrophoretic fractions by previous treatment with the enzyme combinations presented in table 2 showed the presence of radioactive dermatan sulphate, chondroitin 4/6-sul-

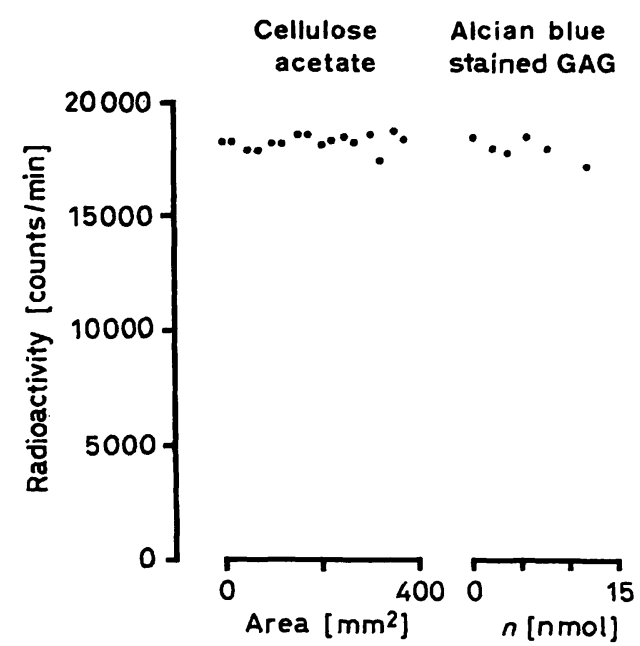

Fig. 2. Influence of cellulose acetate and Alcian Blue on the ${ }^{3} \mathrm{H}$ measurement.

Left diagram: increasing amounts of cellulose acetate were cut out, dissolved in $3 \mathrm{ml}$ of concentrated acetic acid, enriched with $2.22 \mathrm{kBq}(60 \mathrm{nCi})$ of $D$ $\left[{ }^{3} \mathrm{H}\right]$ glucosamine and counted in the presence of $16 \mathrm{ml}$ of Pico-Fluor.

Right diagram: increasing amounts of chondroitin 4sulphate were processed by electrophoresis and stained by Alcian Blue. The fractions, containing increasing amounts of Alcian Blue-stained glycosaminoglycan (GAG) and a constant amount of cellulose acetate, were cut out, dissolved in $3 \mathrm{ml}$ of concentrated acetic acid, enriched with $2.22 \mathrm{kBq}(60 \mathrm{nCi})$ of $D-\left[{ }^{3} \mathrm{H}\right]$ glucosamine and counted in the presence of $16 \mathrm{ml}$ of Pico-Fluor. $n=$ amount of substance

Tab. 2. Indentification of the labelled glycosaminoglycans in the electrophoretic fractions $b$, $c$ and d. Pools of labelled glycosaminoglycan from dermis and epidermis were prepared from skin cultures processed as described in methods sections IV and V, but with twice as much labelled glycosaminoglycans in the electrophoresis sample as usual (labelled glycosaminoglycan/carrier glycosaminoglycan $=$ twice the usual ratio). Each pool was redistributed in 5 volumes of $70 \mu l$ which were incubated at $37^{\circ} \mathrm{C}$ for $8 \mathrm{~h}$ without and with the enzyme combinations presented in the table. Three electrophoretic separations were performed on each volume as described in section VI. The actual counts measured in the fractions are presented in the table. Hence the results are given as counts/min per $20 \mu \mathrm{l}$ of application sample.

\begin{tabular}{|c|c|c|c|c|c|c|c|}
\hline \multirow[b]{4}{*}{ Enzymatic pretreatment } & & \multicolumn{6}{|c|}{$\left[{ }^{3} \mathrm{H}\right]$ Glucosamine-labelled glycosaminoglycan, counts/min } \\
\hline & & \multicolumn{3}{|c|}{ Dermis } & \multicolumn{3}{|c|}{ Epidermis } \\
\hline & & \multicolumn{3}{|c|}{ Electrophoretic fraction } & \multirow{2}{*}{\multicolumn{2}{|c|}{ Electrophoretic fraction }} & \multirow[b]{2}{*}{ d } \\
\hline & & b & c & $\mathrm{d}$ & & & \\
\hline No enzyme & & $\begin{array}{l}2245 \\
2438 \\
2348\end{array}$ & $\begin{array}{l}3117 \\
3524 \\
3305\end{array}$ & $\begin{array}{l}8230 \\
8541 \\
8970\end{array}$ & $\begin{array}{l}811 \\
822 \\
870\end{array}$ & $\begin{array}{l}1857 \\
1909 \\
1853\end{array}$ & $\begin{array}{l}2235 \\
2028 \\
2129\end{array}$ \\
\hline Chondroitinase AC & $(0.33 \mathrm{U})$ & $\begin{array}{l}877 \\
944 \\
878\end{array}$ & $\begin{array}{l}2102 \\
2043 \\
2140\end{array}$ & $\begin{array}{l}27 \\
26 \\
18\end{array}$ & $\begin{array}{l}440 \\
528 \\
481\end{array}$ & $\begin{array}{l}1368 \\
1243 \\
1232\end{array}$ & $\begin{array}{l}24 \\
11 \\
17\end{array}$ \\
\hline $\begin{array}{l}\text { Chondroitinase AC } \\
+ \text { Chondroitinase } \mathrm{ABC}\end{array}$ & $\begin{array}{l}(0.33 U) \\
(0.12 U)\end{array}$ & $\begin{array}{l}135 \\
200 \\
104\end{array}$ & $\begin{array}{l}2525 \\
2556 \\
2595\end{array}$ & $\begin{array}{l}20 \\
41 \\
27\end{array}$ & $\begin{array}{l}134 \\
103 \\
103\end{array}$ & $\begin{array}{l}1478 \\
1496 \\
1470\end{array}$ & $\begin{array}{l}26 \\
24 \\
23\end{array}$ \\
\hline $\begin{array}{l}\text { Chondroitinase AC } \\
+ \text { Chondroitinase ABC } \\
+ \text { Crude heparinase }\end{array}$ & $\begin{array}{r}(0.33 \mathrm{U}) \\
(0.12 \mathrm{U}) \\
(90 \mu \mathrm{g})\end{array}$ & $\begin{array}{l}13 \\
22 \\
15\end{array}$ & $\begin{array}{l}17 \\
26 \\
30\end{array}$ & $\begin{array}{l}0 \\
4 \\
0\end{array}$ & $\begin{array}{l}19 \\
29 \\
18\end{array}$ & $\begin{array}{l}22 \\
31 \\
19\end{array}$ & $\begin{array}{l}1 \\
2 \\
0\end{array}$ \\
\hline $\begin{array}{l}\text { Chondroitinase AC } \\
+ \text { Chondroitinase ABC } \\
+ \text { Keratanase }\end{array}$ & $\begin{array}{l}(0.33 \mathrm{U}) \\
(0.12 \mathrm{U}) \\
(0.25 \mathrm{U})\end{array}$ & $\begin{array}{l}145 \\
188 \\
272\end{array}$ & $\begin{array}{l}2427 \\
2470 \\
2278\end{array}$ & $\begin{array}{l}38 \\
38 \\
25\end{array}$ & $\begin{array}{l}231 \\
156 \\
123\end{array}$ & $\begin{array}{l}1374 \\
1320 \\
1400\end{array}$ & $\begin{array}{r}12 \\
0 \\
25\end{array}$ \\
\hline
\end{tabular}


phate and heparin in fraction $b$, heparan sulphate and chondroitinase AC-susceptible material (compound $\mathrm{X}$ ) in fraction $\mathrm{c}$, and hyaluronic acid in fraction $\mathrm{d}$. Material susceptible to keratanase was not found in any of the fractions. Oddly enough the addition of chondroitinase $\mathrm{ABC}$ reduced the amount of material (compound $\mathrm{X}$ ) removed from fraction $\mathrm{c}$ by chondroitinase AC.

Other experiments showed that band c contained minor amounts of chondroitinase AC-susceptible material not digested by hyaluronidase from Streptomyces (section IX). "Compound X" (Ic-IIIc/Ic-IIc in tab. 1) might therefore be low sulphated chondroitin sulphate, but the final identity of "compound X" remains to be proved.

Our results do not exclude the possibility that minor amounts of keratan sulphate are synthesized. If keratan sulphate is synthesized it should appear in band $\mathrm{d}$ after degradation with chondroitinase and heparinase. The activity after this treatment was small (IIId/ IId in tab. 1) although it differed significantly from the blank values. This small fraction of band $d$ has been called "compound Y" (tab. 1).

The radioactivity of the individual glycosaminoglycan-species was calculated on the basis of the raw data in table 2 . These radioactivity values are presented in the left part of table 3 . Hyaluronic acid was the predominant product of synthesis in both dermis and epidermis, constituting $61 \%$ and $44 \%$ respectively. In epidermis the synthesis of heparan sulphate was almost of the same magnitude $(31 \%)$. The re- maining glycosaminoglycan-species were synthesized at lower rates, each constituting from $1 \%$ (dermal heparin) to $18 \%$ (dermal heparan sulphate) of the synthesized products. On the whole the synthesis in dermis was $2 \frac{1}{2}$ times higher than in epidermis.

Unless it is important to differentiate between the synthesis of dermatan sulphate and the much smaller synthesis of heparin, a less time-consuming method is recommended which only makes use of one of the enzymes: the treatment with chondroitinase AC. This method measures the synthesis of hyaluronic acid, heparan sulphate, "dermatan sulphate" (contaminated with a small fraction of heparin), chondroitin 4/6-sulphate, chondroitinase AC-susceptible material in band $\mathrm{c}$ (compound $\mathrm{X}$ ) and resistant material in $\mathrm{d}$ (compound $\mathrm{Y}$ ). The following experiments were all performed with this simplified method (the method described in detail in the experimental section).

For all glycosaminoglycans concerned, apart from "compound Y", a linear time-dependent incorporation of $D-\left[{ }^{3} \mathrm{H}\right]$ glucosamine was found in both epidermis and dermis during the first 16 hours of labelling (figs. 3 and 4). The incorporation started immediately after addition of the radioactivity. During the first 16 hours the labelling rate of the different glycosaminoglycan species could therefore be compared under equal conditions, i.e. where the rate of synthesis is higher than the rate of degradation. As shown for only hyaluronic acid and heparan sulphate (fig. 5), the proportions of all glycosaminoglycan species remained constant throughout the entire labelling time.

Tab. 3. Synthesis of glycosaminoglycans in skin culture and concentration of in vivo synthesized glycosaminoglycans of uncultured skin. The mean synthesis of glycosaminoglycans in epidermis and dermis was calculated on the basis of the raw data in table 2 using the "A-formulae" of table 1. In addition, glycosaminoglycan from epidermis and dermis of uncultured skin was isolated and separated into species by enzyme treatment and electrophoresis in the same way as labelled glycosaminoglycan. The densitometric scanning areas of the Alcian Blue-stained species were calculated using the "A-formulae". On the basis of a uronic acid measurement of total glycosaminoglycans in epidermis and dermis, the uronic acid concentration in wet skin of each of the epidermal and dermal species was assessed (pmol/mg wet skin). For details see section VIII.

\begin{tabular}{|c|c|c|c|c|c|c|c|c|}
\hline \multirow{3}{*}{ Glycosaminoglycans } & \multicolumn{4}{|c|}{$\left[{ }^{3} \mathrm{H}\right]$ Labeling of glycosaminoglycans } & \multicolumn{4}{|c|}{ Concentration of glycosaminoglycans } \\
\hline & \multicolumn{2}{|l|}{ Dermis } & \multicolumn{2}{|c|}{ Epidermis } & \multicolumn{2}{|l|}{ Dermis } & \multicolumn{2}{|l|}{ Epidermis } \\
\hline & $\begin{array}{l}\text { (counts/ } \\
\text { min) }\end{array}$ & $\begin{array}{l}\text { (per- } \\
\text { centage } \\
\text { of total) }\end{array}$ & $\begin{array}{l}\text { (counts/ } \\
\text { min) }\end{array}$ & $\begin{array}{l}\text { (per- } \\
\text { centage } \\
\text { of total) }\end{array}$ & $\begin{array}{l}\text { (uronic } \\
\text { acid, } \\
\text { pmol/mg } \\
\text { wet skin) }\end{array}$ & $\begin{array}{l}\text { (per- } \\
\text { centage } \\
\text { of total) }\end{array}$ & $\begin{array}{l}\text { (uronic } \\
\text { acid, } \\
\text { pmol/mg } \\
\text { wet skin) }\end{array}$ & $\begin{array}{l}\text { (per- } \\
\text { centage } \\
\text { of total) }\end{array}$ \\
\hline Hyaluronic acid & 8551 & 61 & 2106 & 44 & 243.28 & 47 & 0.48 & 22 \\
\hline Heparan sulphate & 2559 & 18 & 1481 & 31 & 22.25 & 4 & 0.44 & 20 \\
\hline Dermatan sulphate & 753 & 5 & 370 & 8 & 170.30 & 33 & 0.56 & 25 \\
\hline Chondroitin 4/6-sulphate & 1444 & 10 & 351 & 7 & 71.95 & 14 & 0.50 & 23 \\
\hline Compound X (Ic-IIIc, table 1$)$ & 756 & 5 & 392 & 8 & 4.66 & 1 & 0.00 & 0 \\
\hline Compound Y (IIId, table 1) & 29 & 0 & 24 & 0 & 0.00 & 0 & .0 .00 & 0 \\
\hline Heparin & 146 & 1 & 113 & 2 & 5.18 & 1 & 0.22 & 10 \\
\hline
\end{tabular}




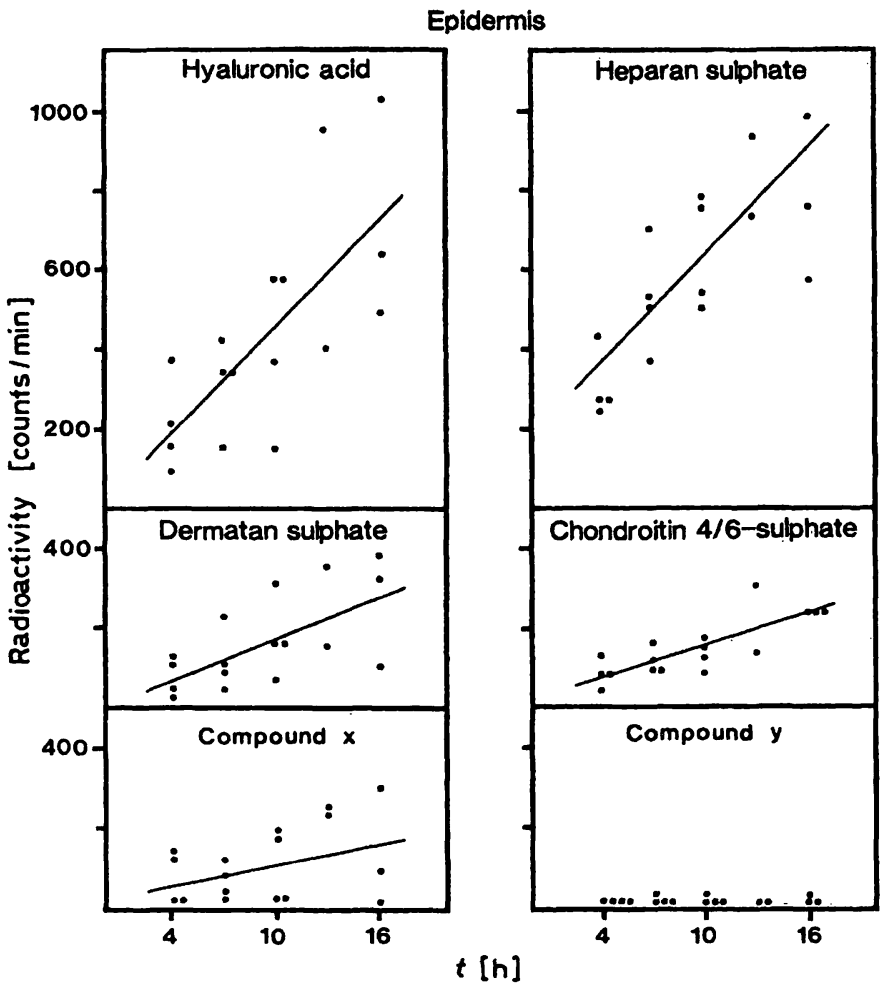

Fig. 3. Time-dependent labelling of glycosaminoglycans in epidermis of cultured skin. Procedures were as described in section IV - VI. Explants, punched from the skin in 4 persons, were cultured with 2 explants in each dish, and labelled for $4,7,10,13$, and $16 \mathrm{~h}$. The radioactivity of the glycosaminoglycan species was measured. Double determinations, made on explants from the same dish, are shown together with the regression line describing the time-labelling relationship (calculated by least squares fit).

The experimental variation was minimized by expressing the epidermal synthesis per explant rather than per mg wet explant, and the dermal synthesis per $\mathrm{mg}$ wet explant rather than per explant (results not shown). The synthesis of glycosaminoglycan-species in 12 explants, punched out of the same piece of skin, was measured (tab. 4). The resulting standard deviations, calculated on a basis of 6 double measurements, demonstrated the overall variation arising from the culture process and from the isolation and separation of the labelled glycosaminoglycans. The greatest variation was found in the epidermal results and, independent of tissue origin, in "compounds $X$ and $Y$ ". No convincing improvement of the precision was gained by expressing the results per $\mu \mathrm{mol}$ of dermal hydroxyproline (tab. 4).

Experiments designed to trace the source of experimental variation were also performed (tab. 5). Half of the glycosaminoglycan-extracts from 12 cultured explants were pooled and redistributed into 6 samples. These samples as well as the 6 unpooled samples were analysed, and the coefficients of variation (CV) from the pooled and unpooled samples were compared.

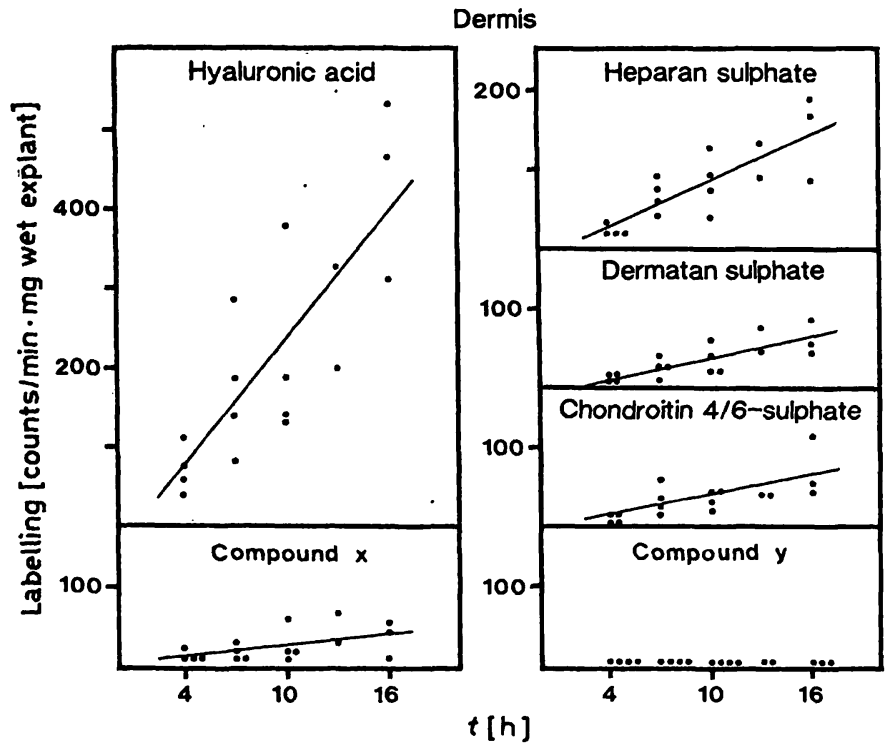

Fig. 4. Time-dependent labelling of glycosaminoglycans in dermis of cultured skin. The experiment is described in tab. 4.

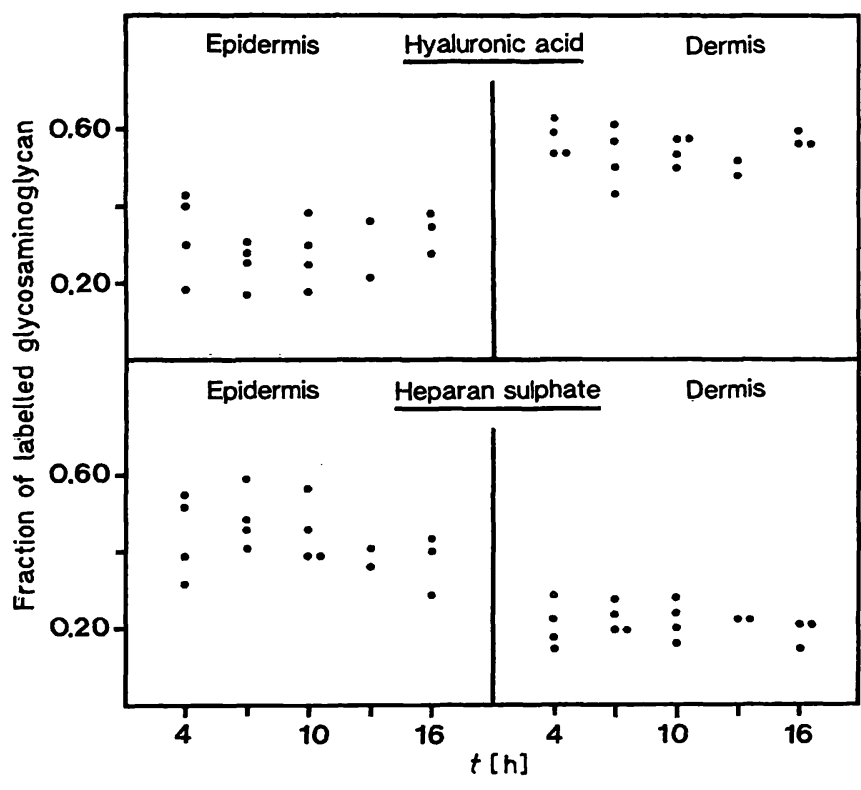

Fig. 5. Proportion of labelled hyaluronic acid and heparan sulphate in skin cultured for 4 to $16 \mathrm{~h}$. The experiment is described in tabs. 4 and 5.

The reduction in $\mathrm{CV}$ produced by pooling the extracts showed that most of the variation in the epidermal measurements arose in the culture process or was due to variation in synthesis within the piece of skin from which the explants had been punched out. The same was true for dermal hyaluronic acid and heparan sulphate. Most of the variation in the measurements of dermal dermatan sulphate, chondroitin $4 / 6$ sulphate and "compound X", on the other hand, arose in the isolation and separation procedures. It is uncertain why the $\mathrm{CV}$ of pooled epidermal samples were lower than the $\mathrm{CV}$ of pooled dermal samples. The 
Tab. 4. Assessment of the experimental variation in the skin culture. Procedure were exactly as described in sections IV - VII. Twelve explants, punched out from the same piece of skin, were cultured with 2 explants in each dish. Labelled glycosaminoglycans were papain-extracted, precipitated by cetyltrimethylammonium bromide and separated by electrophoresis and enzyme pretreatment. The radioactivity of each glycosaminoglycan was calculated using the "B-formulae" table 1 . Originating from samples of $20 \mu \mathrm{l}$, this radioactivity represented $2 / 5$ of the activity occupied by the species in the whole explant. The counts are presented without any corrections (epidermis), as a ratio to the hydroxyproline content in dermis (epidermis, dermis), or as a ratio to the wet weight of the explant (dermis). The results are shown as mean values \pm one standard deviation of 6 double determinations made on explants from the same dish. .

$\left[{ }^{3} \mathrm{H}\right]$ Glucosamine labelling of glycosaminoglycans

Glycosaminoglycans

\begin{tabular}{|c|c|c|}
\hline Epidermis & & \\
\hline counts/min & $\begin{array}{l}\text { counts/min } \\
\mu \text { mol } \\
\text { hydroxy- } \\
\text { proline }\end{array}$ & percent \\
\hline
\end{tabular}

Dermis

counts/min $\cdot$ counts/min $\cdot$ percent mg wet $\quad \mu \mathrm{mol}$ explant hydroxyproline

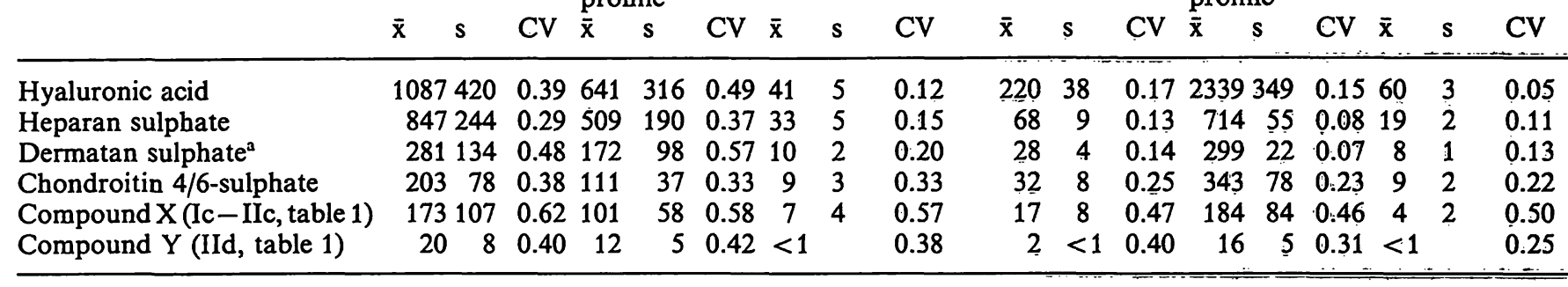

a Contaminated by a small fraction of heparin (for details see text).

Tab. 5. Source of experimental variation in the skin cultures. The experiment described in table 4 was repeated, but this time 6 of the 12 papain digested samples (glycosaminoglycan extracts) were pooled and redistributed in 6 equal volumes before precipitation by cetyltrimethylammonium bromide. The coefficient of variation, based on 6 single determinations, is presented.

\begin{tabular}{|c|c|c|c|c|}
\hline \multirow[b]{3}{*}{ Glycosaminoglycans } & \multicolumn{4}{|c|}{ Coefficient of variation, $\%$} \\
\hline & \multicolumn{2}{|c|}{ Pooled extracts } & \multicolumn{2}{|c|}{ Unpooled extracts } \\
\hline & Dermis & Epidermis & Dermis & Epidermis \\
\hline Hyaluronic acid & 18 & 2 & 51 & 37 \\
\hline Heparan sulphate & 3 & 6 & 19 & 39 \\
\hline Dermatan sulphate & 22 & 8 & 15 & 47 \\
\hline Chondroitin 4/6-sulphate & 31 & 14 & 34 & 20 \\
\hline Compound X (Ic-IIc, table 1) & 82 & - & 80 & - \\
\hline
\end{tabular}

synthesized glycosaminoglycans from both sources were isolated in the presence of surplus carrier glycosaminoglycan of dermal origin. It may be that the epidermal glycosaminoglycans are in a state that enhances their quantitative precipitation.

Sepharose 6B column chromatography demonstrated that the dermal and epidermal material, synthesized in vitro was polymeric, and that it could be degraded by chondroitinase $\mathrm{ABC}+$ heparinase into oligomeric material eluting at the $V_{t}$ (fig. 6). However a small fraction $(2 \%)$ of the glycosaminoglycans synthesized in epidermis still eluted in the void volume after digestion. The significance of this is discussed below. Glycosaminoglycans isolated from dermis which had not been cultured behaved in the same way as the radioactive material in the chromatographic procedures.
For the results presented a complete separation between epidermis and dermis was of course essential. Cross-sections of an entire explant showed that this was actually achieved by the calcium chloride treatment.

In vivo synthesized glycosaminoglycans from uncultured epidermis and dermis

Glycosaminoglycans were isolated from epidermis and dermis of post mortem skin and separated by cellulose acetate electrophoresis. The glycosaminoglycan compositions of the resulting fractions were characterized by experiments with addition of authentic glycosaminoglycans and removal of Alcian Blue positive material from the electrophoresis by previous treatment with chondroitinaise $\mathrm{AC}$, chondro- 

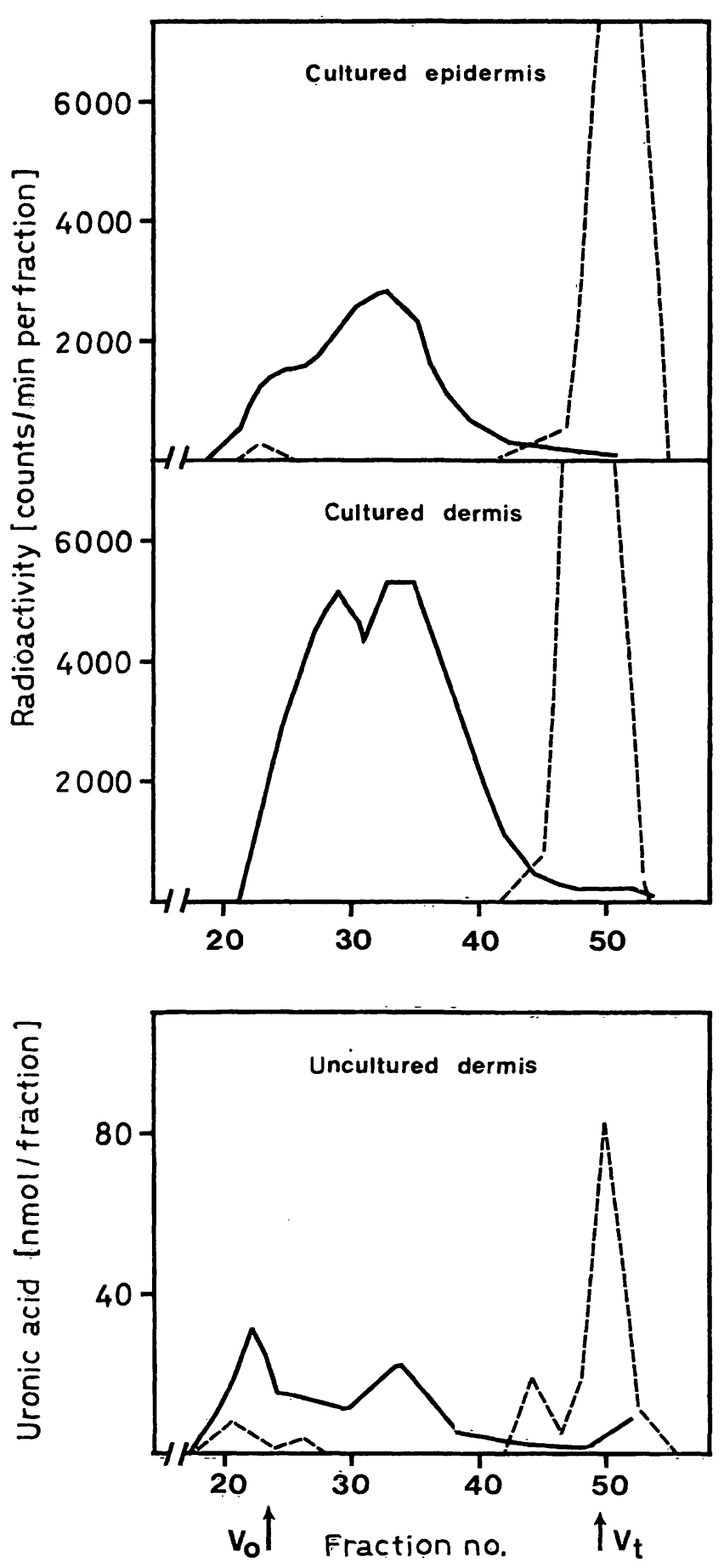

Fig. 6. Sepharose 6B chromatography of glycosaminoglycans from uncultured skin and labelled glycosaminoglycans from cultured skin explants before $(-)$ and after (--) enzymatic digestion. Preparations of labelled glycosaminoglycan were isolated from 16 explants as described in sections IV and V. From these preparations a dermal and an epidermal pool were made. Each pool was divided into two halves which were incubated without and in the presence of chondroitinase $\mathrm{ABC}+$ heparinase at $37^{\circ} \mathrm{C}$ for $8 \mathrm{~h}$ respectively. The solutions were applied to a $0.5 \times 150 \mathrm{~cm}$ Sepharose $6 \mathrm{~B}$ column, and the labelled material was eluted by $0.1 \mathrm{~mol} / 1$ phosphate buffer, $\mathrm{pH} 5.0$, at a flow rate of $2.4 \mathrm{ml} / \mathrm{h}$. Fractions of $0.5 \mathrm{ml}$ were collected and every second fraction was counted in the presence of $5 \mathrm{ml}$ of Pico-Fluor. In vivo synthesized glycosaminoglycan, isolated from dermis of post mortem skin as described in section VIII, was chromatographed in the same way, and monitored as uronic acid. itinase $\mathrm{ABC}$ and heparinase: Fraction $\mathrm{b}$ consisted of chondroitin 4/6 sulphate, dermatan sulphate and heparin-like glycosaminoglycans. Fractions c contained mainly heparan sulphate, susceptible to heparinase, but also material susceptible to chondroitinase $A C$ or $\mathrm{ABC}$ (compound $\mathrm{X}$ ). Both hyaluronic acid and keratan sulphate migrated with fraction d. However it was completely removed by chondroitinase $\mathrm{AC}$ and fraction $\mathrm{d}$ was hence identified as hyaluronic acid.

Successive removal of glycosaminoglycan species from the electrophoretic fractions by enzymes made it possible to determine the uronic acid concentration in whole skin of 6 epidermal and 6 dermal glycosaminoglycans. The results were compared with the synthesis figures in table 3 . More than $99 \%$ of the skin glycosaminoglycans were found in dermis. The predominant constituents of dermis were hyaluronic acid and dermatan sulphate, constituting $47 \%$ and $33 \%$ respectively, whereas dermal chondroitin $4 / 6$ sulphate, heparan sulphate and heparin constituted only $14 \%, 4 \%$ and $1 \%$. In epidermis the proportions of heparan sulphate and heparin were much higher, constituting $20 \%$ and $10 \%$ respectively. Epidermal hyaluronic acid, dermatan sulphate and chondroitin 4/6sulphate constituted $22 \%, 25 \%$ and $23 \%$. It is evident that these tissue concentrations do not reflect the rates by which the glycosaminoglycans are synthesized in the explants. The significance of this is discussed below.

\section{Discussion}

The labelled products in the skin explants were identified as glycosaminoglycan on the basis of different characteristics: the products resisted digestion by papain, deoxyribonuclease and ribonuclease. They complexed with cetyltrimethylammonium bromide at $\mathrm{pH} 5$ and were eluted from a Sephrose 6B column as macromolecules like native dermal glycosaminoglycans. In cellulose acetate electrophoresis the labelled products migrated with authentic glycosaminoglycans and native glycosaminoglycan from dermis and epidermis. After treatment with chondroitinase $A B C$ plus heparinase, the labelled materials were eluted from the Sepharose column as small molecules and became undetectable by cellulose acetate electrophoresis.

However, some $2 \%$ of the labelled material still appeared in the void volume when labelled epidermal glycosaminoglycans were eluted after digestion (fig. 6). We do not believe that this residue is the result of an insufficient proportion of enzyme in the digest, or the presence of any inhibitors. When glycosaminoglycan preparations of all kinds other than keratan 
sulphate were added to the incubate they were completely degraded under the conditions used. The undigested material from epidermis may be either keratan sulphate or contaminating cell surface associated glycoconjugates which are known to also incorporate $\left[{ }^{3} \mathrm{H}\right]$ glucosamine $(4,8)$. Glycoconjugates of low charge density may very well appear in the void volume and be absent from the electrophoretic zones of glycosaminoglycans. This was in fact what was seen in our results.

Dermatan sulphate, including dermatan sulphate from pig skin as an example, is a copolymer with chondroitinase AC-susceptible disaccharide units of chondroitin 4-sulphate (11). Digestion by chondroitinase AC however did not change the mobility or stainability of dermatan sulphate from porcine skin when electrophoresed on cellulose acetate. The dermatan sulphate oligosaccharides produced in this way maintain mobility and stainability of the parent dermatan sulphate. Hence there is no reason to believe that the low labeling rate of dermatan sulphate was due to loss of dermatan sulphate oligosaccharides.

The present model uses skin biopsies which includes epidermis and the entire dermis and measures the synthesis of glycosaminoglycans in both layers. As papain treatment extracts both cellular and extracellular glycosaminoglycans from the tissue (8) the model does not distinguish between these sources.

Labelled glycosaminoglycans may be lost in the calcium chloride solution used to separate epidermis from dermis. This was the case when the separation was performed by trypsin or dispase treatment (4), but according to King \& Tabiowo (4) the adopted separation procedure, using calcium chloride incubation, was superior to the enzyme methods with respect to this source of error.

As in all culture experiments the same batch of foetal calf serum should be used for comparative studies, and whenever possible one should culture two ex- plants from each individual, as most of the experimental variation arose "before" isolation and separation of the labelled glycosaminoglycan. By expressing the radioactivity per $\mu \mathrm{mol}$ of dermal hydroxyproline no improvement in precision was gained. The experimental variation was minimized by expressing the epidermal counts "per explant" and the dermal counts "per mg of explant".

A labelling time of 16 hours ensured that the incorporation-time relationship had not reached a plateau for any of the glycosaminoglycan species. The labell= ing rate of the species could therefore be compared under equal conditions.

The labelling rate of glycosaminoglycan was $2 \frac{1}{2}$ times higher in dermis than in epidermis. This difference could hardly be explained by differences in the number of cells between the two layers, although the dermal number exceeded that of the epidermis by $15 \%$ to $70 \%$ (range of cell counts in four cross sections).

Hyaluronic acid, heparan sulphate, dermatan sul= phate, chondroitin 4/6-sulphate, heparin, and a chondroitinase AC-susceptible fraction with the mobility of heparan sulphate (compound $\mathrm{X}$ ) were demonstrated as in vivo constituents in both dermis and epidermis. The same species were also synthesized there in vitro. However, there was no proportionality between the tissue concentrations and the in vitro synthesis rates. In dermis the concentration of dermatan sulphate equalled the concentration of hyaluronic acid, and considerably exceeded the concentration of heparan sulphate. Nevertheless the dermal synthesis of dermatan sulphate was much smaller than the dermal synthesis of both hyaluronic acid and heparan sulphate. Likewise the synthesis of hyaluronic acid and heparan sulphate were very predominant in the epidermal synthesis, but no single glycosaminoglycan species predominated in the in vivo synthesized glycosaminoglycan pool of uncultured epidermis.

Tab. 6. The relative turnover rate of glycosaminoglycan species assessed by in vitro and in vivo investigations. The turnover rate of dermatan sulphate is used as a unit of measurement. For further explanation see "Discussion".

\begin{tabular}{|c|c|c|c|c|}
\hline & \multirow{2}{*}{\multicolumn{2}{|c|}{$\frac{\text { In vitro synthesis }}{\text { Tissue concentration }}$}} & \multirow{3}{*}{$\begin{array}{l}\frac{\text { Urinary excretion }^{\mathrm{a}}}{\text { Tissue concentration }} \\
\text { Dermis }\end{array}$} & \\
\hline & & & & \\
\hline & Epidermis & Dermis & & \\
\hline Hyaluronic acid & 7 & 8 & not excreted & \\
\hline Heparan sulphate & 5 & 26 & 10 & " \\
\hline Dermatan sulphate & 1 & 1 & 1 & \\
\hline Chondroitin 4/6-sulphate & 1 & 5 & no correlation & \\
\hline Heparin & 1 & 6 & not measured & \\
\hline
\end{tabular}

a Poulsen, J. H. \& Wæth, M. (1984) Scand. J. Clin. Lab. Invest. 44, 535-539. 



\section{Fllavins and Flavoproteins 1987}

\section{Proceedings of the Ninth International Symposium Atlanta, Georgia, U.S.A., June 7-12, 1987}

\section{Editors D. E. Edmondson - D. B. McCormick}

$1988.17 \mathrm{~cm} \mathrm{X} 24 \mathrm{~cm}$. XXIV, 775 pages. Numerous illustrations. Hardcover. DM 360,-; approx. US \$225.00 ISBN 3110109506

This volume contains contributed papers presented at the 9th International Symposium on Flavins and Flavoproteins held in June 1987 at Emory University, Atlanta, Georgia, U.S.A.

A broad range of investigations on the structure and mechanism of flavoenzymes, structure and electron transfer mechanisms, and biosynthesis and medical aspects are presented.

This book contains the most recent compilation of research efforts and views on the biological function of vitamin $\mathrm{B}_{2}$ (flavins). Approaches used in the contributions range from $x$-ray crystallography and NMR spectroscopy to gene cloning techniques.

Contents (Main Chapters)

Structure and Mechanisms of Flavoenzymes - Flavoenzyme Structure and Electron Transfer Mechanisms · Flavin Biosynthesis, Metabolism and Medical Aspects . Flavoenzyme Hydroxylases · Flavin-Dependent Bioluminescence · Structure, Mechanism and Biosynthesis of Flavoenzymes Containing Covalently-Bound Flavins - List of Participants · Author Index · Subject Index.

\section{Also available}

\section{Flavins and Flavoproteins}

Proceedings of the Eighth International Symposium

Brighton, England, July 9-13, 1984

Editors R. C. Bray · P. C. Engel -S. G. Mayhew

1984. $17 \mathrm{~cm} \times 24 \mathrm{~cm}$. XXXIV, 923 pages. Numerous illustrations. Hardcover. DM 285,-; approx. US \$ 178.00 ISBN 3110098792 
On the basis of the results in table 3 we therefore calculated the ratio between the labelling rate and the tissue concentration of glycosaminoglycan for each species (tab. 6). If the in vitro synthesis reflects the pattern of synthesis in vivo, this ratio is directly proportional to the rate by which the pool of a certain glycosaminoglycan species is replaced at steady state. This turnover was much higher in epidermis than in dermis, probably because the greater part of the glycosaminoglycans in dermis, unlike the glycosaminoglycans in the cell-rich epidermis, is so far from the cells that they only participate in the turnover to a limited extent (in the intact skin).

To our knowledge it has not been shown before that heparin-like glycosaminoglycan is present in a relative large amount in epidermis, and that heparin is synthesized there. In this report heparin or heparin-like glycosaminoglycan however was defined somewhat arbitrarily as heparinase-susceptible and chondroitinase $\mathrm{ABC}$-resistant glycosaminoglycan migrating with heparin. No attempts were made to characterize this fraction by its protein core structure or by its anticoagulant activity (12). Hence it may be that this heparin fraction only represents some highly sulphated heparan sulphate.

Endothelial cells also produce heparin-like substances which in vitro have been shown to stimulate the synthesis of glycosaminoglycan by arterial smooth muscle cells while markedly decreasing the proliferation of these cells $(13-14)$. It is possible that the heparin-like substances in epidermis have a similar effect in the epithelial-mesenchymal relationship of skin.

In table 6 we have also presented results from an earlier in vivo investigation (16). On the basis of a direct proportionality between the urine excretion and the dermal concentration of heparan sulphate and dermatan sulphate, it was tempting to calculate the ratios between corresponding urine and tissue measurements as an attempt to estimate the relative turnover of the two glycosaminoglycans in the dermis. The metabolic fate of heparan sulphate and dermatan sulphate is hardly the same, and the urine-tissue ratios are probably inaccurate or may be even misleading measures of the in vivo turnover. Hence it may be pure chance that these figures show the same pattern as the ratios between the in vitro synthesis and the tissue concentration of heparan sulphate and dermatan sulphate (tab. 6).

\section{Acknowledgement}

We thank Carl Chr. Danielsen, Institute of Anatomy, University of Aarhus, DK-8000 Aarhus C, Denmark for making the histological cross-sections.

\section{References}

1. Muir, H. \& Hardingham, T. E. (1975) MTP Int. Rev. Sci. Biochem. Ser. I. 5, 153-222.

2. Dietrich, C. P. (1984) Brazilian J. Med. Biol. Res. 7, 5-15.

3. Reid, M. L. \& Rojkind, M. (1979) In: Methods in Enzymology: Cell Culture (Jakoby, W. B. \& Pastan, I. H., eds.) Vol. 58, pp. 263-278, Academic Press, New York.

4. King, I. A. \& Tabiowo, A. (1980) Biochim. Biophys. Acta. $632,234-243$.

5. Anseth, A. (1971) Exp. Eye Res. 11, 251-254.

6. Cremer-Bartels, G. \& Buddecke, E. (1972) Exp. Eye Res. $14,171-172$.

7. Klintworth, G. K. \& Smith, C. F. (1976) Lab. Invest. 35, $258-263$.

8. King, I. A. (1981) Biochim. Biophys. Acta 674, 87-95.

9. Blumenkrantz, N. \& Asboe-Hansen, G. (1973) Anal. Biochem. 54, 484-489.
10. Blumenkrantz, N. \& Asboe-Hansen, G. (1974) Clin. Biochem. 9, 269-274.

11. Fransson, L. $\AA$ (1968) Biochim. Biophys. Acta 156, $311-$ 317.

12. Gallagher, J. T., Lyon, M. \& Steward, W. P. (1986) Biochem. J. 236, 313-325.

13. Hoover, R. L., Rosenberg, R., Haering, W. \& Karnovsky, M. J. (1980) Circ. Res. 47, 578-583.

14. Castellot, J. J., Addonizio, M. L., Rosenberg, R. \& Karnovsky, M. (1981) J. Cell. Biol. 90, 372-379.

15. Harris, S. A., Gajdusek, C., Schwartz, S. M. \& Wight, T. N. (1981) Fed. Proc. 40, 623.

16. Poulsen, J. H. \& Væth, M. (1984) Scand. J. Clin. Lab. Invest. $44,535-539$.

Jørgen Hjelm Poulsen, M. D., Dr. med. Department of Clinical Chemistry University of Aarhus

Aarhus Kommunehospital

DK-8000 Aarhus C 
